\title{
A New Educational Program to Improve Posture and Quality of Life Among Students by Means of the Feldenkrais Method and a New Program: MAP-Motion and Posture
}

\author{
Iris Gil \\ Correspondence: Iris Gil, Ohalo College of Education; Shaanan Academic Religious Teachers College; Kaye Academic \\ College of Education, 211 HaEfroni St., Atzmon-Segev, Misgav, Israel.
}

Received: June 28, 2018

doi:10.11114/jets.v6i11.3582
Accepted: August 22, $2018 \quad$ Online Published: August 29, 2018

URL: https://doi.org/10.11114/jets.v6i11.3582

\begin{abstract}
Newborns possess a natural ability for correct body posture, but most of us lose this ability to the extent of causing damage and consequently a decline in quality of life. Damage can be manifested in pain (mainly in the back), decline in functioning, and damage to emotional aspects of life. To examine the nature of the connection between body posture and quality of life by focusing on the question of whether and how practicing the well-known Feldenkrais method or the unknown Motion and Posture (MAP) method improves quality of life and body posture. The study was conducted among 243 students. An educational program for correct posture was conducted with physical exercises derived from both the Feldenkrais method and the new MAP method. The study employed a mixed-methods approach, and the tools utilized were two questionnaires, a digital inclinometer, Visual Analog Scale (VAS), and a metric tape measure. A positive trend of improvement was found both in the participants' quality of life and posture. The qualitative measurement found a connection between improvement in quality of life and improvement in posture. The participants in the MAP program had an advantage over the participants in the Feldenkrais program. The innovation of the present study is in presenting the importance of posture for quality of life. This study is a new construct for understanding quality of life as a synergy between emotional and physical aspects that draws on improving posture by means of the Feldenkrais and MAP methods.
\end{abstract}

Keywords: quality of life, posture (of the body), feldenkrais method, MAP (Motion and Posture)

\section{Introduction}

\subsection{Literature Review}

\subsubsection{Quality of Life}

Throughout history, various definitions have been proposed for the expression "the common good", such as "happiness" and "the good life" (George \& Bearon, 1981), and over the past few decades, "quality of life". The extensive literature engaging with research on quality of life reveals that a clear and consistent general definition does not exist for the term, and its origin is unknown (Bandura, 2011). Since the end of the twentieth century attempts have been made to define quality of life in different social contexts (family, happiness, cultural, economic). One of the contexts in which the largest amount of research has been carried out regarding "quality of life" is in the field of health.

\subsubsection{Health Promotion}

Formulation of health promotion theories began in recent years. This includes reaching out to the general public and offering instructions on how to increase participation in activities such as physical exercise that promote good health and quality of life in order to prevent diseases and improve both physical and mental health (Murray, Rodgers \& Fraser, 2012).

Another relevant perception of quality of life lies with Dr. Moshe Feldenkrais (1904-1984). According to Feldenkrais, an individual can have a high quality of life when he has physical and mental unity, though chances are that this unity will break due to various life situations. To preserve this balance and improve the individual's wellbeing, Feldenkrais treated movement as a means for improving other components of wellbeing, such as insight, sensitivity, and emotions (Feldenkrais, 1967; Feldenkrais, 1976; Feldenkrais, 1989). 
In the present study the term "quality of life" can be considered as follows: on the one hand, the quality of human life comprises the individual's current subjective perception of his different life aspects (health, emotional) and of the environment in which he lives, and his past and future expectations; and on the other, it comprises the individual's ability to maintain inner unity between all the parts of his being (movement, insight, senses, and emotion) (Gil, 2013).

The extensive professional and academic literature engaging with the connection between quality of life and postural defects shows that correct body posture is one of the essential indices for the human body to function in harmony (Chaikin-Bentor, 2016; Limiolek, 2008).

\subsubsection{Correct Posture}

There are three body systems that are directly connected to movement and posture: the nervous system that is responsible for all our bodily activities and is the one that sends commands to the muscles regarding what, how, to what extent, and when to carry them out; the muscular system that moves the bones in the body; and the skeletal system that includes the bones and the joints between the bones (Gil, 2013).

Correct posture development occurs in an ordered and consistent manner from infancy to maturity, and is similar in the majority of human beings. In the same way, a postural "disorder" leading to postural defects is also a process that develops in similar ways in most people.

Lack of harmony where posture is concerned can lead to a number of problems (Schnake \& Kandziora, 2010). For example, shortening of the lower back muscles can also cause limitation in the diaphragm muscles, which are an integral part of the normal breathing process (Solberg, 2013).

Posture Impairments

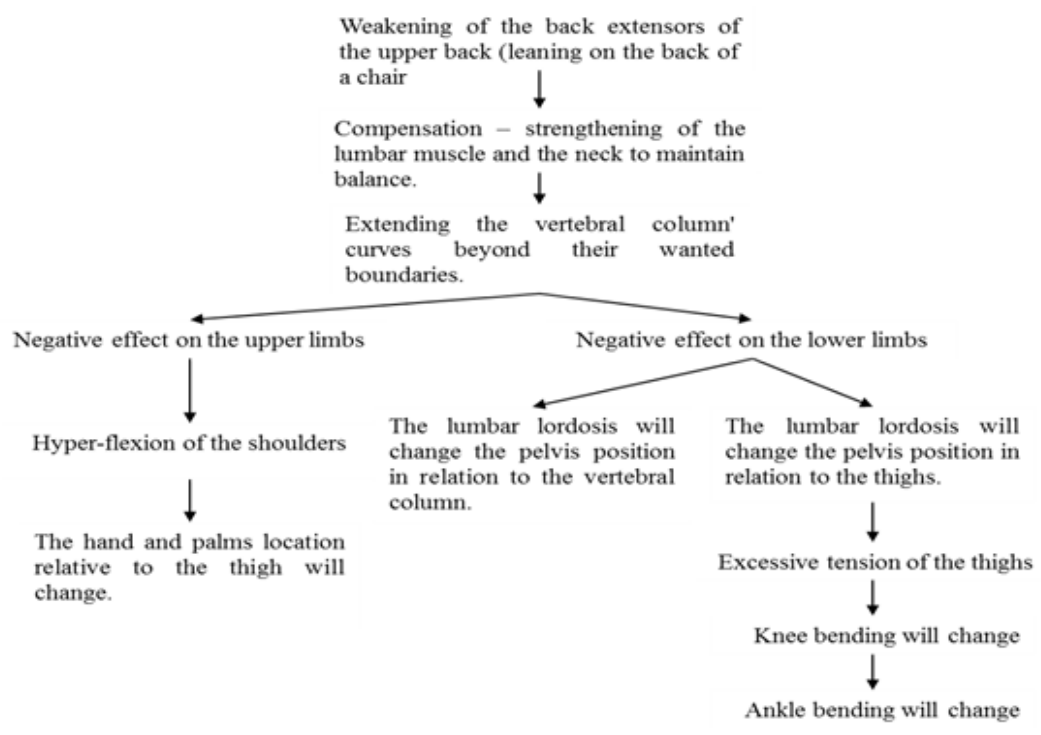

Figure 1. Impairment Development Sequence

Figure 1 indicates that incorrect use of the back muscles is at the "top of the pyramid" where responsibility for posture impairment is concerned. This incorrect use is found in many people primarily in the extension and weakening of their upper back muscles (from T1 to T12) - kyphosis - and in compression and shortening of their lower back muscles (from L1 to L5) - lordosis (Gil, 2013; Lamartina, 2010; Youdas, Garrett, Egan \& Therneau, 2010).

Both these impairments are the focal points of reference and consideration in the physical educational examinations carried out in the present study.

The present study examined the connection between posture and quality of life by means of two educational programs to improve posture.

(1) Feldenkrais: Dr. Moshe Feldenkrais believed that people are born with correct natural movements, but over the years they adapt wrong, and even harmful, movement habits. This can gradually be corrected by developing body awareness (Feldenkrais, 1967).

According to the educational Feldenkrais method, movement is the practical way to improve quality of life. In light of this, Dr. Moshe Feldenkrais initiated hundreds of motility classes that include a number of principles:

- Neutralizing gravity - most of the exercises are performed in unconventional physical positions that neutralize gravity (such as lying on the back, or in other ways). These positions create changes in relation to gravity, thus enabling 
the muscles to "disregard" their customary activity and to contract, thus providing a new way to work in a completely different neuromuscular manner.

- Much repetition of every exercise.

- Gentle movements that reduce muscular tension.

- Using different variations of the same exercise.

- Creating a relaxed atmosphere in the class.

(2) Dr. Feldenkrais formulated an innovative educational method based on philosophical and practical-physical approaches (Feldenkrais, 1967).

According to his method, improving and perfecting motility results in posture improvement. Improvement and perfection of these abilities are contingent on neural and behavioral changes that occur when exercising in unique gentle techniques.

Other, more conventional methods for posture improvement, such as physiotherapy, are based on strengthening certain posture muscles: abdominal, pelvic, and back (Kloubec, 2010; Ligia, 2011; Sarig, 2002). Accordingly, control over the pelvic floor provides the basis for organizing posture, which in turn improves functional abilities, links body and mind, and leads to improvement of quality of life.

In order to combine the advantages of the Feldenkrais method with more conventional methods, the MAP (Motion and Posture) program was created.

(3) MAP: This program is both similar to and differs from the Feldenkrais method in many aspects. The practical lessons, as well as the guidance and educational process accompanying them, are all adapted from the Feldenkrais method. However, the MAP program utilizes Feldenkrais method lessons that possess one of the following two characteristics: lessons that are taught in body positions that contend with gravity, such as sitting, standing, and walking, and lessons taught while lying down but using muscles from the body center, such as the back, stomach, and pelvis. These are the muscles that are responsible for body posture in accordance with conventional methods (Oliveira, Liebano, Costa LCM, Rissato \& Costa LOP, 2013).

The rationale behind these choices was that strengthening the posture muscles, as well as exercising and guidance in different body positions, will allow the advantages of the Feldenkrais method to be "copied" and preserved for use in everyday life when certain muscles need to contend with gravity.

The literature shows that employing the Feldenkrais method improves quality of life (Netz \& Lidor, 2003). Up until 2011 there was no evidence of a connection between implementing the Feldenkrais method and posture improvement. Therefore, this study was created.

\subsection{The Present Study}

\section{The aim of the study}

To examine the nature of the connection between body posture and quality of life.

\section{The questions of the study}

1. Whether and how does practicing the Feldenkrais method improve quality of life and body posture?

2. Whether and how does practicing the MAP method (a combination of Feldenkrais and strengthening of posture muscles) improve quality of life and body posture?

\section{The hypothesis of the research}

Hypothesis 1: The participants' quality of life - both their physical status (back, movement) and emotional state (mood) - will improve.

Hypothesis 2: The participants' general posture and spinal column curvature angles (e.g., kyphosis and lordosis) will improve.

Hypothesis 3: Positive correlation will be found between posture indices (kyphosis and lordosis) and quality of life indices (physical status and emotional state).

Hypothesis 4: Differences in quality of life and posture will be found between participants exercising in the Feldenkrais method in comparison with participants exercising in the MAP method. 


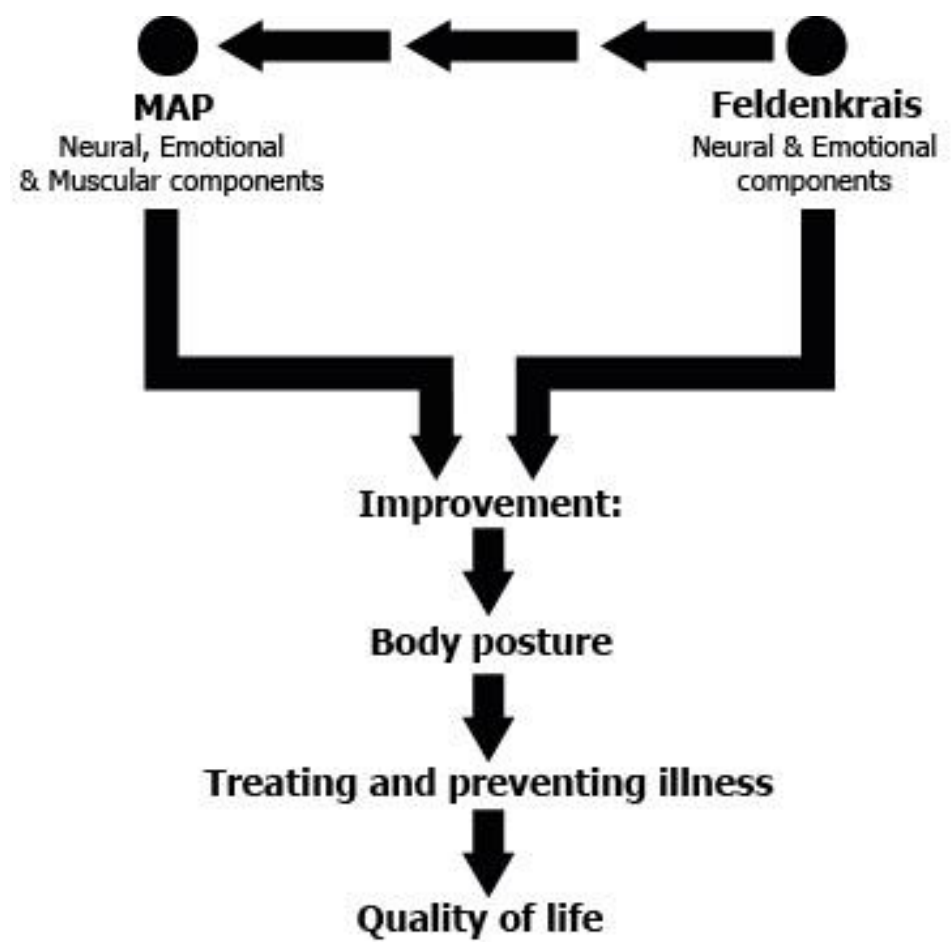

\section{Method}

Figure 2. The Theoretical Model

\subsection{Research Approach}

The aim of the present study was to enrich existing knowledge on the connection between "correct posture" and "quality of life". Since the research was carried out during the participants' regular professional studies, a pragmatic paradigm was the correct fit for the study. Therefore, a mixed-methods research approach was chosen since it combines a quantitative approach and tools with a qualitative approach and tools. The quantitative tools employ accurate physical examinations to test the effects of educational programs on posture improvement. The qualitative tools allow an understanding of the processes the participants experienced during the study, enabling the qualitative tools to enrich the quantitative ones. The quantitative research consists of an experimental part, and the qualitative research emphasizes action research (Creswell, 2009).

The strategy of this mixed-methods research was Sequential Explanatory Design, a popular and typical mixed-methods strategy, which is used when the quantitative research is primary, and the qualitative data are secondary. Its goal is to explain and interpret the quantitative outcome by collecting and analyzing qualitative data (Bryman, 2006; Creswell, 2009).

\subsection{Research Stages}

The research comprised two parts. The first part took place during the 2011/2012 winter semester, and the second during the spring of 2012.

Due to technical difficulties, some of the data collected in the first part were unusable. Additionally, some changes occurred between the two parts that were related to the research tool and improvement in the examiners' skills. Therefore, the second part of the research constitutes its main part.

All the participants (243) in both parts underwent three stages:

Stage 1: At the beginning of the research the participants completed a quality of life questionnaire, and spinal curvature measurements were taken.

Stage 2: During the research, the participants took part in an intervention program (14 meetings/lessons). Half of them took part in a Feldenkrais program and half in a MAP program.

Stage 3: At the end of the research, the same tests were carried out as in Stage 1.

Additional tests were carried out in the second stage on some of the participants: spinal curvature measurements before and after one lesson, height measurements before and after one lesson, recording the participants' mood (on a scale of 1-10) at the end of five lessons, and participation in the qualitative part of the study. 


\subsection{Participants}

The participants were 243 students for B.A. from the technological institute "Technion" "at Haifa, Israel at the aged 18-28 (average age 24), from different faculties. 70\% of the students were female. The students examined at 2011-2012 during their sequential studies.

Table 1. Participants

\begin{tabular}{ccc}
\hline Group Type & Number of Students & Number of research participants \\
\hline Feldenkrais & $\mathbf{1 6 0}$ & $\mathbf{1 1 1}$ \\
MAP & $\mathbf{1 6 0}$ & $\mathbf{1 3 2}$ \\
\hline
\end{tabular}

\subsection{Research Tools}

\subsubsection{Quantitative Measurements}

SrS-22 - Quality of Life Questionnaire: Quality of life was measured by means of the SrS-22 questionnaire (from Health Status Measure). The questionnaire comprises 22 items relating to back and spine health and how they affect quality of life. Participants were requested to rate each question on a 5-point Likert scale.

VAS (Visual Analog Scale): Participants were asked to rate their general mood at the end of five random lessons during the semester. Mood was measured on a Likert scale, with responses ranging from 1 (extremely low) to 10 (extremely high) (Shye, 1989).

Digital Inclinometer: The vertebral column was measured by two sub-measures at each measuring point: (a) kyphosis was measured in an upper (C7) and lower (T12) point using a digital inclinometer, after which an absolute gap was calculated between these points; and (b) lordosis was measured in an upper (T12) and lower (L5) point using a digital inclinometer, after which an absolute gap was calculated between these points as well (Le Huec, Saddiki, Franke, Rigal \& Aunoble, 2011).

These measures were taken pre-intervention, post-intervention, and in a single class in the middle of the semester.

Metric Tape Measure: Height difference was measured during one random lesson, and was calculated between the beginning and end of the lesson (in millimeters) (Kloubec, 2010).

The reliability of the quantitative measurements based on an objective study and high odds for similar results to exist in repeating studies. The Cronbach's alpha average coefficient was 0.8 for those quantitative measurements.

\subsubsection{Qualitative Measures}

The Lessons Effect Questionnaire: The open questionnaire was prepared specifically for this study and contained five open questions that enabled the participants to respond as they saw fit, describing the processes they underwent in the educational program, and how it affected them.

The questions were:

1. What were your emotions at the end of the class?

2. When did those feeling fade and you felt 'back to normal'?

3.Did you learn or experienced anything new in the class?

4. What did you 'take' from the class to your everyday life?

5. Do you feel a cumulative effect of the classes on your everyday life?

In order for a scientific nature to be attributed to the participants' responses, an encoding process was initiated every few stages. In the initial stage, the participants' responses to each question in the questionnaire were sorted and reorganized in a different order. The reorganization involved a statement describing one of the following single categories on its own: feeling, thought, sense and movement. These categories comprise the main components of the Feldenkrais method and similarly, of the MAP method.

Restoring the participants' responses according to categories provided the foundation for the second stage, in which the statements provided below each category were taken and reorganized into themes.

First theme: Improvement effects of the single lesson

Second theme: Effects of all the educational programs on improved posture

Third theme: Improvement of quality of life as a cumulative effect of posture improvement. 
In the third stage, each theme was divided into a number of categories. After providing a heading for each category and relating the category to the research hypotheses, the relevant question was presented under this heading, in the same way as in the qualitative questionnaire. These categories allowed for confirmation or rejection of the research hypotheses.

The reliability of the qualitative measures was the researcher herself because it is difficult to expect that in the case of reusing the "Lessons Effect" questionnaire, other researchers will receive the same results. The variance between the participants is huge and there is almost no possibility that another will express insights, emotions, behaviors and senses from the lesson as another. In the attempt to increase reliability, few steps were taken:

1. Creating a data base presented at all times to other researchers, not only a concluding report.

2. Saving the analysis documents by 3-4 stages. Every stage and its documents and protocols, categories of analysis and their content. Other colleagues-researchers may use the data: to trace what was done and promote additional analysis.

\section{Data Analysis}

In order to test differences between groups and along measurement points over time, repeated measures analysis of variance (ANOVA) was conducted. The variance was tested in relation to two groups: (1) Group difference - examining the differences between the group that learned the MAP program and the participants who learned the Feldenkrais program; and (2) Difference in time period - examining the difference between the participants' initial status prior to the intervention program and their status at the end of the program. In order to test correlations between variables a Pearson test was conducted.
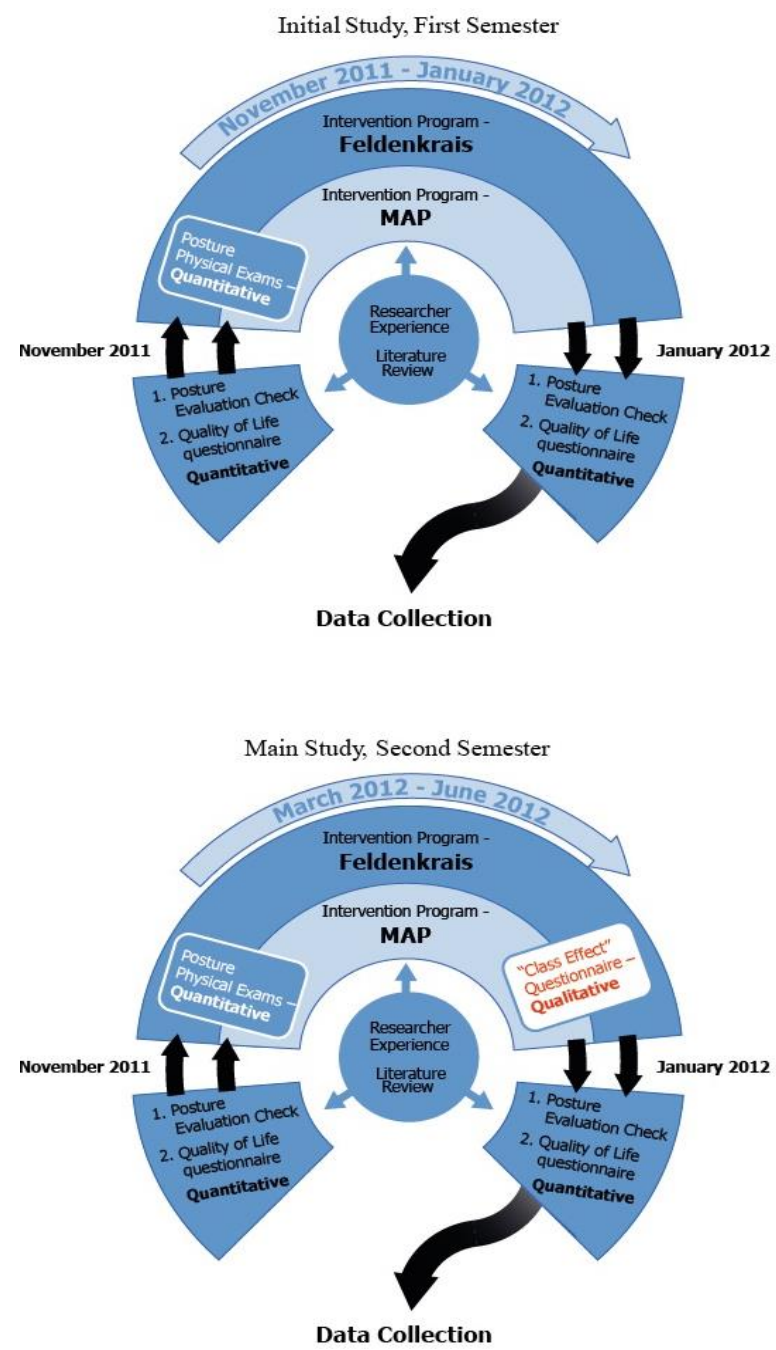

Figure 3. The Research Process 


\section{Results}

\subsection{Quantitative Analysis}

Hypothesis 1: The participants' quality of life - both their physical status (back, movement) and emotional state (mood) - will improve.

\section{SrS-22 - Quality of Life Questionnaire}

A repeated measures procedure was conducted on the entire sample. The independent variable in this analysis was group type (Feldenkrais vs. MAP), and the dependent variable was quality of life.

Table 1 shows descriptive statistics of general quality of life and sub-domains of quality of life in the groups.

Table 2. Sub-Domains of Quality of Life and General Quality of Life

\begin{tabular}{lllll}
\hline Measure & \multicolumn{2}{c}{ Pre-intervention } & \multicolumn{2}{c}{ Post-intervention } \\
\hline & Feldenkrais (N=39) & MAP $(\mathrm{N}=100)$ & Feldenkrais (N=39) & MAP (N=100) \\
Back Condition & $3.75(0.74)$ & $3.83(0.77)$ & $3.90(0.76)$ & $3.97(0.71)$ \\
Emotional Condition & $3.64(0.47)$ & $3.54(0.48)$ & $3.83(0.49)$ & $3.79(0.57)$ \\
Physical Condition & $3.87(0.57)$ & $3.85(0.53)$ & $3.89(0.55)$ & $3.89(0.53)$ \\
General Quality of Life & $3.63(0.41)$ & $3.54(0.44)$ & $3.69(0.46)$ & $3.65(0.49)$ \\
\hline 2 shows that general & quality of life improved among participants in both groups between pre- and
\end{tabular}

Table 2 shows that general quality of life improved among participants in both groups between pre- and post-intervention.

VAS (Visual Analog Scale)

In order to explore differences in mood improvement, the mood of some of the participants was measured immediately following the end of five lessons. A repeated measures procedure was conducted on the entire sample. The independent variables were the participants, and the dependent variables were mood measures.

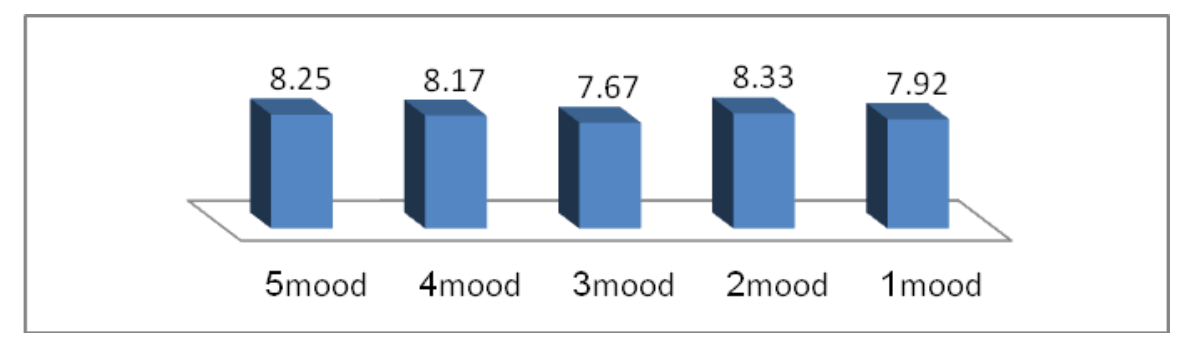

Figure 4. Differences in Mood Measures Over Five Sessions

Figure 4 indicates no significant differences between mood measures during these five lessons.

Hypothesis 2: The participants' general posture and spinal column curvature angles (e.g., kyphosis and lordosis) will improve.

Digital Inclinometer

Pre- and post-intervention program sample: In order to examine Hypothesis 2, a repeated measures procedure was conducted on the entire sample. The independent variable in this analysis was group type (Feldenkrais vs. MAP), and the dependent variables were the absolute gaps in kyphosis and lordosis between pre- and post-intervention.

Table 2 shows the descriptive statistics of gaps in kyphosis and lordosis between pre- and post-intervention by groups.

Table 3. Gaps in Kyphosis and Lordosis between Pre- and Post-Intervention

\begin{tabular}{lllll}
\hline Measure & \multicolumn{2}{c}{ Pre-intervention } & \multicolumn{2}{c}{ Post-intervention } \\
\hline & Feldenkrais (N=69) & MAP $(\mathrm{N}=54)$ & Feldenkrais (N=69) & MAP (N=54) \\
Kyphosis gap & $20.55(7.87)$ & $14.66(7.18)$ & $16.36(7.11)$ & $15.76(8.29)$ \\
Lordosis gap & $9.35(8.40)$ & $18.98(8.26)$ & $6.97(4.89)$ & $12.18(7.84)$ \\
\hline
\end{tabular}

Table 3 shows that in general, the gap in kyphosis was unchanged in participants from both groups between pre- and post-intervention.

Regarding lordosis, results showed that among participants from both groups lordosis decreased (improved) between pre- and post- intervention.

Digital Inclinometer Pre- Lesson and Post-Single Lesson 
In order to examine differences in spinal column measures following a single lesson, a repeated measures procedure was conducted on part of the sample. The independent variable in this analysis was group type (Feldenkrais vs. MAP), and the dependent variables were absolute gaps in kyphosis and lordosis between the beginning and end of the lesson.

Table 4. Gaps in Kyphosis and Lordosis- Single Lesson

\begin{tabular}{lllll}
\hline Measure & \multicolumn{2}{c}{ Beginning of session } & \multicolumn{2}{c}{ End of session } \\
\hline & Feldenkrais $(\mathrm{N}=42)$ & MAP $(\mathrm{N}=35)$ & Feldenkrais $(\mathrm{N}=42)$ & M.A.P $(\mathrm{N}=35)$ \\
Kyphosis gap & $16.52(8.89)$ & $17.54(10.17)$ & $16.16(8.43)$ & $15.20(9.54)$ \\
Lordosis gap & $11.00(9.46)$ & $13.82(11.94)$ & $11.68(9.43)$ & $12.08(10.60)$ \\
\hline
\end{tabular}

Table 4 shows that the gaps in kyphosis and lordosis in a single lesson were unchanged among participants from both groups.

\subsection{Metric Tape Measure}

In order to expand and reinforce the quantitative and physical findings obtained with the digital inclinometer, the height of some of the participants was also measured at the beginning and end of a lesson.

In order to examine this hypothesis, an analysis of variance (ANOVA) procedure was conducted. The dependent variable was height difference, and the independent variable was group type.

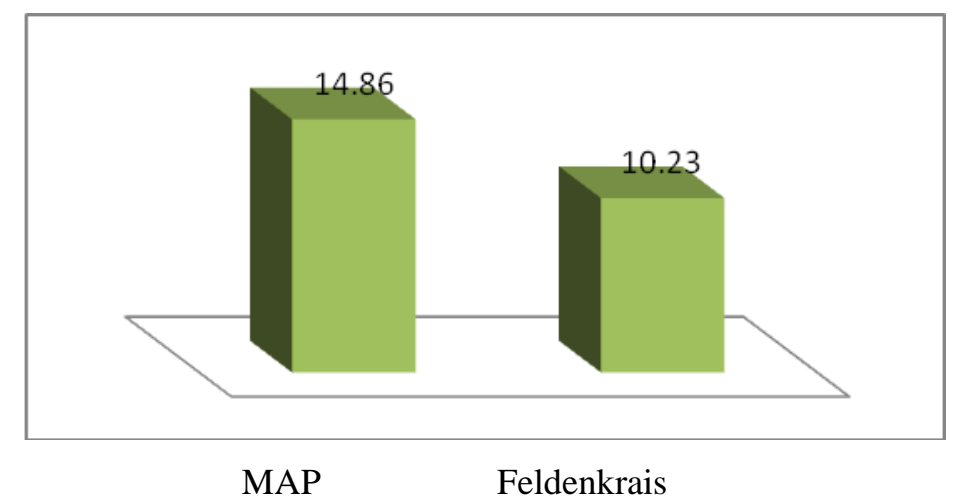

Figure. 5. Height Differences - Single Lesson

Figure 5 shows some improvement in height; however, this finding should be treated with caution since not many participants took part in this test.

Hypothesis 3: A positive correlation will be found between posture indices (kyphosis and lordosis) and quality of life indices (physical status and emotional state).

In order to examine this hypothesis, Pearson correlations were produced between improvement in kyphosis/lordosis and quality of life measures, as presented in Table 4.

Quality of Life

Table 5. Quality of Life and Posture by Group

\begin{tabular}{|c|c|c|c|c|c|c|c|c|}
\hline & \multicolumn{2}{|c|}{$\begin{array}{c}\text { General Quality of } \\
\text { Life }\end{array}$} & \multicolumn{2}{|c|}{ Back Condition } & \multicolumn{2}{|c|}{$\begin{array}{c}\text { Emotional } \\
\text { Condition }\end{array}$} & \multicolumn{2}{|c|}{ Physical Condition } \\
\hline & Feldenkrais & MAP & Feldenkrais & MAP & Feldenkrais & MAP & Feldenkrais & MAP \\
\hline $\begin{array}{l}\text { Kyphosis } \\
\text { Gap }\end{array}$ & . 100 & .061 & .002 & .586 & .067 & .050 & .173 & .096 \\
\hline $\begin{array}{l}\text { Lordosis } \\
\text { Gap }\end{array}$ & .063 & .224 & .083 & .111 & .057 & .183 & .200 & .150 \\
\hline
\end{tabular}

As presented in Table 5, no significant correlations were found between improvement in kyphosis or lordosis and quality of life measures.

Hypothesis 4: Differences in quality of life and posture will exist among participants exercising in the Feldenkrais program in comparison with participants exercising in the MAP program. 
Table 6. Quality of Life Sub-domain Gaps in the Groups

\begin{tabular}{lcc}
\hline Measure & Feldenkrais $(\mathbf{N}=\mathbf{3 8})$ & MAP $(\mathbf{N}=\mathbf{1 0 0})$ \\
\hline Back Condition & $0.26(0.38)$ & $0.41(0.34)$ \\
Emotional Condition & $0.19(0.19)$ & $0.35(0.28)$ \\
Physical Condition & $0.16(0.31)$ & $0.24(0.23)$ \\
\hline
\end{tabular}

Despite the impressive results regarding quality of life in both groups, Table 6 shows that no significant difference was found between the gaps in both groups.

Posture

Kyphosis (between pre- and post-intervention): While no difference was found in the kyphosis gap among participants in the MAP program, the kyphosis gap decreased and improved among participants in the Feldenkrais program.

Lordosis (between pre- and post-intervention): Participants in the MAP program showed greater improvement in lordosis gaps.

\subsection{Qualitative Analysis}

The first theme (the beneficial effects of a single lesson) includes all the participants' responses that describe the immediate effects that a particular lesson had on them.

The second theme (the effects of all educational posture programs) presents an analysis of the participants' responses regarding the change and improvement they experienced following all educational posture improvement programs.

The third theme (improvement of quality of life as a cumulative effect of posture improvement) presents the perception of Dr. Moshe Feldenkrais who analyzed the term "quality of life" based on four components: motion, insight, sense, and emotion.

Many of the responses regarding improvement and cumulative changes referred to physical states, but also to quality of life components. Therefore, there is an evident trend of improving quality of life by improving posture, as stated in Hypothesis 3.

\section{Conclusions}

\subsection{Conclusions Regarding Improving Quality of Life}

The findings show significant improvement in the participants' quality of life due to both posture improvement programs, and thus the factual conclusion is that quality of life can be improved by means of the Feldenkrais and MAP educational movement programs.

From the perspective of existing literature, the method expands the literature in two aspects: the connection between (physical and mental) health and quality of life, and the positive connection between the Feldenkrais method and quality of life.

\subsection{Conclusions Regarding Improving Posture}

The findings showed a significant improvement in the participants' posture in both groups. Thus, the factual conclusion is that posture can be improved by participating in the studied educational posture improvement programs: Feldenkrais and MAP.

The importance of this conclusion is that it corroborates existing literature regarding the connection between physical activity and posture improvement, and the connection between exercising in the Feldenkrais method and improvement in quality of life. It also expands the accumulating knowledge base regarding Feldenkrais as a method that also improves physical measures by means of exercise or using gentle movement techniques. The innovation of the present conclusion is that for the first time proof has been found of a connection between a certain amount of exercise according to a familiar method - Feldenkrais - and exercising with a novel program - MAP - and posture improvement. The significance is that by relating to the body as a whole unit (rather than just to the back or the legs separately, as is the norm), and by relating to the nervous system (not only the muscular system, as is the norm), posture can be improved.

\subsection{The Correlation Between Improving Posture and Improving Quality of Life}

The point of departure of this hypothesis was that if posture and quality of life significantly improve due to the posture improvement programs, a positive correlation will be found. In fact, this hypothesis was confirmed by analyzing the qualitative findings as expressed in the Lesson Effect Questionnaire, but was not confirmed by the statistical analysis.

Since the two findings for the same hypothesis differ, the conclusions should be treated with caution. The findings constitute a developing trend that needs further examination. 


\subsection{The Innovation of the Study}

The innovation is presentation of the importance of posture for quality of life. This study is a new construct for understanding quality of life as a synergy between emotional and physical aspects that draws on posture improvement through a pedagogical physical activity program incorporating Feldenkrais and MAP.

\subsection{Recommendations}

\subsubsection{General Applicable Recommendations}

1. Encourage the general public to acknowledge the importance of moderated physical activity for improving quality of life and preventing posture impairments.

2. The Feldenkrais method and the MAP program should be further examined for implementation in other populations: younger or older populations, more intellectual normative populations and populations with special needs, both intellectual and emotional.

3. The programs should be recommended for several public bodies: the health system, the education system and the business sector.

\subsubsection{Recommendations for Further Research}

1. Is there a connection between emotional situations or certain personality traits and posture, and if so, what is it?

2. Can posture also be improved with the help of the Feldenkrais and MAP methods in dynamic and changing situations, such as walking?

3. Can learning ability be improved using the Feldenkrais and MAP methods?

4. Do any other quality of life and posture improvement programs use movement? Can more be created?

5. What is the connection between the Feldenkrais and MAP methods and additional physical measurements, such as balance?

\subsection{The Limitations of the Research}

Regarding the participants: During signup for the research, there was lack of details and data concerning the participants, for instance, their previous or additional physical activity. In addition, the level of their physical activity was not noted before and beyond the research.

Regarding the researcher stance: The fact that the researcher herself was teaching the program lessons, and the fact that the researcher was the conductor of the research, it might have been a factor in the students' will or discomfort.

Regarding the scientific process: No follow up was conducted for the research results beyond the research period, as in the time frame after the research.

\section{References}

Bandura, A. (2011). A social cognitive perspective on positive psychology. Rev. Psychologies Social, 26, 7-20. https://doi.org/10.1174/021347411794078444

Bryman, A. (2006). Mixed Programs: A four-volume set. London: Sage. https://doi.org/10.4135/9781446262566

Chaikin-Bentor, N. (2016). Correct movement, optimal posture and walking. Narkisim, Israel.

Creswell, J. W. (2009). Research design (3rd ed.) Lincoln, Nebraska: University of Nebraska Press.

Feldenkrais, M. (1967). Practical ability improvement. Tel Aviv: The Feldenkrais Institute.

Feldenkrais, M. (1976). Chapters in my method. Tel Aviv: Aleph Publishing.

Feldenkrais, M. (1989). Body and mature behavior. Tel Aviv: The Feldenkrais Institute.

George, L. K., \& Bearon, L. B. (1981). Quality of life in older persons: Meaning and measurement. PsycCRITIQUES, 26(4), 307-308.

Gil, I. (2013). A new educational program for improving posture and quality of life among students by using the Feldenkrais and MAP methods. (2013). Doctoral dissertation, Wingate Institute for Physical Education and Sports, Israel; 2013.

Kloubec, J. A. (2010). Pilates for improvement of muscle endurance, flexibility, balance and posture. Journal of Strength and Conditioning, 24(3), 661-667. https://doi.org/10.1519/JSC.0b013e3181c277a6

Lamartina, C. (2010). Posterior surgery in Scheuermann's kyphosis. European Spine Journal, 19(3), 515-516. https://doi.org/10.1007/s00586-010-1351-8 
Le Huec, J. C., Saddiki, R., Franke, J., Rigal, J., \& Aunoble, S. (2011). Equilibirium of the human body and the gravity line: The basics. European Spine Journal, 20, 558. https://doi.org/10.1007/s00586-011-1939-7

Ligia, M. P., Karen, O., \& Josiliainne, M. D. (2011). Comparing the Pilates method with no exercise or lumbar stabilization for pain and functionality in patients with chronic low back pain: Systematic review and meta-analysis. Clin. Rehabil, 26(1), 10-20.

Lmiolek, M. (2008). Spine mobility and the quality of body posture in 11-year old handball players compared to their peers. Journal of Sport Medicine, 5(6), 24.

Murray, T. C., Rodgers, W. M., \& Fraser. S. N. (2012). Exploring the relationship between socioeconomic status, control beliefs and exercise behavior: A multiple mediator model. Journal of Behavioral Medicine, 35(1), 63-73. https://doi.org/10.1007/s10865-011-9327-7

Netz, Y., \& Lidor, R. (2003). Mood alterations in mindful versus aerobic exercise modes. Journal of Psychology, 137, 405-419. https://doi.org/10.1080/00223980309600624

Oliveira, R. F., Liebano, R. E., Costa, L. C. M., Rissato, L. L., \& Costa, L. O. P. (2013). Immediate effects of region-specific and non-region-specific spinal manipulative therapy in patients with chronic low back pain: A randomize controlled trial. Physical Therapy, 93(6), 748-756. https://doi.org/10.2522/ptj.20120256

Sarig, Y. (2002). A collection of lectures. The National Training Center for Bone Building. Tel Aviv, Israel.

Schnake, K. J., \& Kandziora, F. (2010). Correction of posttraumatic kyphosis of the thoracolumbar spine with modified pedicle subtraction osteotomy. European Spine Journal, 19(12), 2231-2232. https://doi.org/10.1007/s00586-010-1622-4

Shye, S. (1989). The systemic life quality model: A basis for urban renewal evaluation. Social Indicator Research, 21(4), 343-378. https://doi.org/10.1007/BF00303952

Solberg, G. (2013). Posture and movement: Postural disorders and musculoskeletal dysfunction: Diagnosis, prevention and treatment (4th ed). Independently published, Israel.

Youdas, J. W., Garrett, T. R., Egan, K. S., \& Therneau, T. M. (2010). Lumbar lordosis and pelvic inclination in adults with chronic low back pain. Physical Therapy, 80(3), 261-275.

\section{Copyrights}

Copyright for this article is retained by the author(s), with first publication rights granted to the journal.

This is an open-access article distributed under the terms and conditions of the Creative Commons Attribution license which permits unrestricted use, distribution, and reproduction in any medium, provided the original work is properly cited. 